\title{
BMJ Open Health-related quality of life after pulmonary embolism: a cross-sectional study
}

Mazdak Tavoly, ${ }^{1,2,3}$ Kristin Kornelia Utne, ${ }^{1,2}$ Lars-Petter Jelsness-Jørgensen, , ,4 Hilde Skuterud Wik, ${ }^{5}$ Frederikus A Klok, ${ }^{6}$ Per Morten Sandset, ${ }^{2,4}$ Waleed Ghanima ${ }^{1,2}$

To cite: Tavoly M, Utne KK, Jelsness-Jørgensen L-P, et al. Health-related quality of life after pulmonary embolism: a cross-sectional study. BMJ Open 2016;6: e013086. doi:10.1136/ bmjopen-2016-013086

- Prepublication history for this paper is available online To view these files please visit the journal online (http://dx.doi.org/10.1136/ bmjopen-2016-013086).

Received 19 June 2016 Revised 11 September 2016 Accepted 30 September 2016

\section{(1) crossmatk}

${ }^{1}$ Department of Medicine, Østfold Hospital Trust, Fredrikstad, Norway ${ }^{2}$ Institute of Clinical Medicine University of Oslo, Oslo, Norway

${ }^{3}$ Department of Medicine, Sahlgrenska University Hospital, Gothenburg, Sweden

${ }^{4}$ Department of Health Science, Østfold University College, Fredrikstad, Norway ${ }^{5}$ Department of Haematology, Oslo University Hospital Rikshospitalet, Oslo, Norway ${ }^{6}$ Department of Thrombosis and Hemostasis, Leiden University Medical Center, Leiden, The Netherlands

Correspondence to Dr Mazdak Tavoly; mazdak.tavoly@vgregion.se

\section{ABSTRACT}

Objectives: The psychological effects of acute pulmonary embolism (PE) have scarcely been studied. The aims of this study were to evaluate health-related quality of life (HRQoL) in patients with a history of $P E$ compared with that of the general population and buddy controls, and to explore factors that may predict impaired HRQoL.

Design: Cross-sectional.

Setting: Haematology and thrombosis unit in Fredrikstad, Norway.

Participants: 213 consecutive patients treated for PE were identified from hospital registries. Eligible patients were scheduled for a single study visit, including a functional capacity test (6 min walking test). HRQoL was assessed using the EuroQol 5D dimensions 3-level (EQ-5D-3L) questionnaire, of which the results were compared with Danish population norms and agematched and sex-matched buddy controls. The buddy controls were recruited by asking every patient to hand over the EQ-5D questionnaire to 2 age-matched and sex-matched friends or relatives. Multivariable regression analyses were used to examine possible determinants of reduced HRQoL.

Results: Mean age was 61 years (SD 15), 117 (55\%) were males, and median time since diagnosis was 3.8 years (range 0.3-9.5). Mean EuroQol visual analogue scale (EQ VAS) was 67 in PE as compared with 81 in the general population $(p<0.005)$ and corresponding EQ-5D index values were 0.80 and 0.86 $(p<0.005)$. Patients reported more problems in all 5 EQ-5D compared with both the buddy controls and the general population, $p<0.05$. Shorter 6 min walking distance $(\beta=0.09, p<0.005)$ and patient-reported dyspnoea $(\beta=11.27, p<0.005)$ were independent predictors of lower EQ VAS scores.

Conclusions: Our findings show that patients with a history of PE have impaired HRQoL when compared with the general population and buddy controls. Reduced functional capacity and persistent dyspnoea were the main predictors of this impairment.

\section{INTRODUCTION}

Health-related quality of life (HRQoL) after deep vein thrombosis (DVT) has been

\section{Strengths and limitations of this study}

- This study describes the long-term health-related quality of life, functional capacity and prevalence of dyspnoea in patients with a history of pulmonary embolism, which have scarcely been studied.

- A large sample size in which all aspects of a generic health-related quality of life questionnaire is reported combined with functional capacity assessment.

- The findings of this study may encourage future studies to evaluate the susceptibility of these patients to cardiopulmonary rehabilitation.

- The low response rate and the retrospective design may hamper external validation.

extensively studied. The interest in studying HRQoL in DVT is believed to be related to the well-characterised frequent detrimental chronic condition of post-thrombotic syndrome (PTS) that affects $30-50 \%$ of patients with DVT. ${ }^{1}$ Unlike DVT, long-term effects of acute pulmonary embolism (PE) on HRQoL are understudied. The equivalent long-term complication of acute PE is chronic thromboembolic pulmonary hypertension $(\mathrm{CTEPH}){ }^{2}$ This condition has been shown to affect 2$4 \%$ of the patients with a history of PE. ${ }^{3}$ This relatively low frequency of CTEPH may be the reason for the limited number of studies focusing on HRQoL and the psychological well-being of patients with PE. ${ }^{4-9}$ It has been suggested that CTEPH itself is the extreme manifestation of a much more common phenomenon of permanent changes in pulmonary haemodynamics, cardiac function and pulmonary gas exchange after acute $\mathrm{PE}$, which is associated with dyspnoea and decreased exercise capacity. Additionally, several studies have shown that up to $50 \%$ of the patients with a history of PE report persistent dyspnoea a long time after PE. ${ }^{4}{ }^{10}$ By analogy with PTS after DVT, it was recently 
proposed to refer to this phenomenon as the 'postpulmonary embolism syndrome'. ${ }^{11}$ Moreover, a recent Scandinavian study reported the overuse of antidepressants in adolescents with a history of PE, indicating that PE may develop into a chronic illness in a relevant number of patients. ${ }^{12}$ Indeed, the few existing studies all report an impaired HRQoL in patients with a history of PE compared with that of the normal population, ${ }^{4} 6$ although the results concerning possible predictors of reduced HRQoL are divergent. ${ }^{7}$ A more detailed knowledge of the determinants of HRQoL is needed to allow for identification of treatment targets and implementation of this important end point in future outcome studies.

The aims of this study were to compare HRQoL in patients with a history of PE to that of the general population and age-matched and sex-matched controls, and to evaluate possible determinants of HRQoL.

\section{MATERIALS AND METHODS}

Participants and setting

Consecutive patients who were diagnosed and treated for PE at the Østfold Hospital Trust, Fredrikstad, Norway, between January 2002 and December 2011 were identified from the hospital's registries including the thrombosis registry by searching for International Classification of Diseases (ICD)-10 codes of PE (ICD-10 I26.0 and I26.9). All patients alive at the beginning of March 2012, and with a PE diagnosis confirmed by CT pulmonary angiogram or high probability perfusion scintigraphy, were eligible for study participation.

Patients were excluded if they were aged $<18$ or $>90$ years or deemed incapable of complying with study procedures, including language barriers, geographical unavailability, known dementia, psychiatric diagnosis such as major depression as well as affective disorders or any degree of psychotic disorder. Patients living in nursing homes or receiving major help from social care services were excluded as well.

Written informed consent was obtained for all patients.

\section{Study design}

All eligible patients were contacted by telephone and invited to participate in the study. Patients who responded to our invitation were scheduled for a visit during which they underwent physical examination and functional capacity test using the 6 min walking test. The 6 min walking test is a standardised functional capacity test, which is widely used to objectively assess patients' cardiopulmonary capacity. ${ }^{13}$ The test was performed according to published guidelines, ${ }^{14}$ by one of the study investigators (MT). For each patient, we derived predicted values from the recommendations of the literature. ${ }^{15}$ Evaluation of patients comprised blood tests including brain natriuretic peptide (BNP), which were obtained at the study visit. Sociodemographic data were recorded on standardised case record forms.

Prior to the study visit, the HRQoL questionnaire was sent to the patients either by email or post. Patients were asked to complete the form at home and return it at the scheduled study visit. Incomplete forms were completed during the visit at the hospital.

\section{Quality of life questionnaire}

The validated Norwegian version of the EuroQol fivedimension three-level (EQ-5D-3L) questionnaire was used in order to assess quality of life (QoL). EQ-5D-3L consists of a descriptive system and the EuroQol visual analogue scale (EQ VAS). ${ }^{16}{ }^{17}$ The EQ-5D-3L is a validated, generic, preference-based, health status measure consisting of five descriptive questions encompassing five domains of HRQoL: mobility, self-care, usual activities, pain/discomfort and anxiety/depression. Each question is answered based on three response options (1='no problems', 2='moderate problems', 3='severe problems'). The $243\left(3^{5}\right)$ potential patterns of responses each indicate a unique health state ranging from 11111 for perfect health to 33333 for the worst possible state. The health states can then be converted into a single summary index value, which ranges from 1 (state of full health) to values lower than 0 (states regarded as worse than being dead).

The EQ VAS is a self-rated health on a vertical visual analogue scale $(0-100)$ where the end points are labelled 'worst imaginable health state' and 'best imaginable health state'.

\section{Control groups}

Although several European countries have established normative population data for the EQ-5D instrument, these are not available for Norway. Therefore, we compared our results to the Danish population norms that were established in 2009. ${ }^{18}$

To correct for incident cases with venous thrombosis in the normative population, we included a second control group by asking our study participants to recruit two age-matched ( \pm 5 years) and sex-matched relatives or friends without a history of venous thrombosis, hereafter referred to as buddy controls, to complete the EQ-5D form. Buddy controls were asked to return the anonymous questionnaire in prepaid envelopes. Owing to the anonymity of the buddy controls, further baseline characteristics were not accessible.

\section{Predictors}

On the basis of clinical experience and previous research, we hypothesised that the following determinants may be relevant predictors of HRQoL after PE: (1) age, (2) sex, (3) disease duration (time in years from PE diagnosis to study visit), (4) body mass index $\left(\mathrm{kg} / \mathrm{m}^{2}\right)$, (5) recurrent venous thromboembolism, (6) occupation, (7) persistent patient-reported dyspnoea, (8) performance at 6 min walking test, (9) BNP, (10) 
active malignancy, (11) ongoing anticoagulant treatment, (12) known cardiopulmonary comorbidity, including interstitial pulmonary diseases, congestive heart failure and chronic obstructive pulmonary disease and (13) proximal clot location at PE diagnosis as assessed by a previously published radiological score by Ghanima et $a l^{19}$

\section{STATISTICAL ANALYSES}

Continuous variables were expressed as means and SDs if normally distributed and as medians with ranges if the distribution was skewed. Categorical variables were presented as percentages and/or frequencies. Comparisons were made using Student's t-test or Mann-Whitney U test (depending on normal or skewed distribution) for continuous variables and $\chi^{2}$ tests for categorical variables.

Since very few patients and controls had 'extreme problems', the EQ-5D were dichotomised to either 'no problems' or 'problems'.

Age and gender adjustment of controls was made by weighing the population norm EQ-5D index values and EQ VAS with the distribution of our sample, as recommended by Hjermstad et al. ${ }^{20}$

Variables deemed predictive of HRQoL were first screened using univariate analysis (Spearman's $r$ ). Correlations below the significance level of $\alpha=0.1$ were retained for the multivariate regression analysis. Potential multicollinearity was checked before inclusion in the multivariate models. Then multivariate regression analyses comprising both standard linear regression and binary logistic regression models were performed. For the former, the possible determinants were tested for independency against EQ VAS and $r^{2}$ was used to estimate the percentage of effect explained by the model. For the latter, retained determinants from the univariate analysis were tested for independency against each of the EQ-5D dimensions. The Hosmer and Lemeshow test was used to estimate the goodness of fit of the model.

The multiple imputation model was used in order to deal with missing values in the EQ-5D questionnaires of the buddy controls in whom we did not have the possibility to check and complete the questionnaires during a study visit. ${ }^{21}$ Cases with more than $50 \%$ of the items or EQ VAS missing were omitted. All analyses were performed using the Statistical Package for Social Science V.22.0 (SPSS, Chicago, Illinois, USA), and considered significant at a two-sided $\alpha$ of $\leq 0.05$.

\section{RESULTS}

\section{Study flow}

A total of 836 patients were identified and assessed for eligibility in this study. As shown in the study flow chart (figure 1), 430 (51\%) patients were excluded according to the predefined exclusion criteria. Of the 406 remaining and thus invited patients, 189 (46\%) declined to participate. Of the remaining 217 eligible patients, 213 completed both the EQ-5D questionnaire and

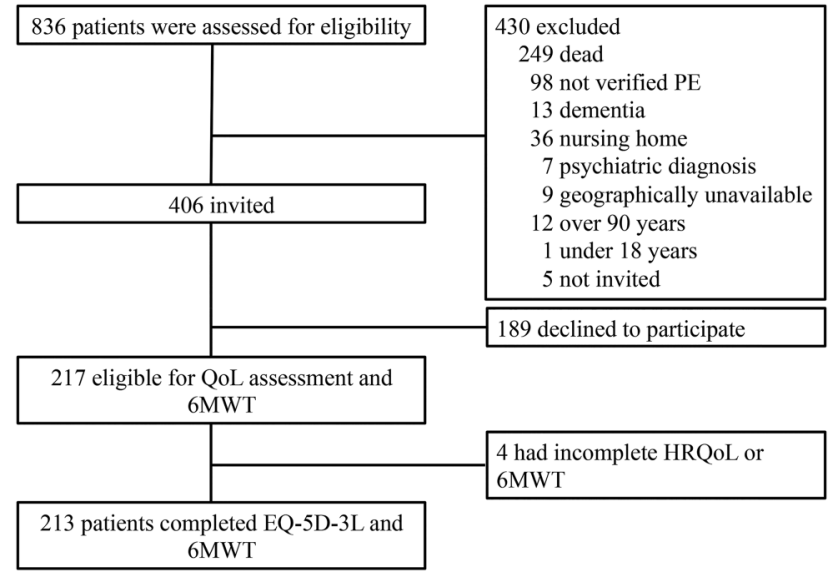

Figure 1 Study flow chart. 6MWT, 6 min walking test; EQ-5D-3L, EuroQol five-dimension three-level; HRQoL, health related quality of life; PE, pulmonary embolism; QoL, quality of life.

underwent the 6 min walking test. Hence, the response rate for our study cohort was $52 \%$.

The number of buddy controls who returned the EQ-5D form was 205, of whom 28 returned questionnaires had more than $50 \%$ of data missing. After excluding these 28, 177 were left for analysis. The response rate for the buddy controls was thus $42 \%$, assuming all study patients indeed forwarded the questionnaire to two 'buddies'.

\section{Study patients}

Patients had a mean age of 61 years (SD 15) and 55\% were men $(n=117)$. Sociodemographic characteristics are presented in table 1 . Median time since diagnosis was 3.8 years (range $0.3-9.5$ ) with $89 \%$ being diagnosed with PE more than a year prior to study inclusion.

Mean distance covered on the 6 min walking test by the study cohort was $449 \mathrm{~m}$ (SD 135). The mean 6 min walking distance was $97 \mathrm{~m}$ (95\% CI 76 to 117) less in male patients and $84 \mathrm{~m}$ (95\% CI 65 to 104) less in female patients as compared with their gender-predicted value, $\mathrm{p}<0.005$.

\section{Comparison of HRQoL between patients, population controls and buddy controls}

Table 2 shows the frequency of reported problems by dimension as well as mean and median values for $\mathrm{EQ}$ VAS and EQ-5D index values stratified by age group. The dimensional difference between patients and both of the control groups yielded statistically significant differences across all dimensions (figure 2). Comparisons of EQ-5D index values and EQ VAS between patient and control groups are presented in table 3. A comparison with the male proportion of our sample to that of the Danish population norms regarding EQ-5D index values initially showed a statistically significant difference, $\mathrm{p}=0.04$ (0.84 vs 0.88 ). However, after adjusting for outliers, the statistical significance disappeared ( 0.85 vs $0.87, \mathrm{p}=0.13$ ). 
Table 1 Sociodemographic and clinical characteristics of the study sample

\begin{tabular}{ll}
\hline Variable & Study sample \\
& n (\%) \\
\hline Female & $96(45)$ \\
Age in years, mean (SD) & $61(15)$ \\
Years since diagnosis, median (range) & $3.8(0.3-9.5)$ \\
Occupation & \\
Unemployed & $50(24)$ \\
Working & $71(33)$ \\
Retired & $92(43)$ \\
Diagnosis & \\
PE & $149(70)$ \\
PE+DVT & $64(30)$ \\
Recurrent VTE & $34(16)$ \\
Cardiopulmonary comorbidity & $19(9)$ \\
BMI, mean (SD) & $28.7(4.9)$ \\
Obesity & $73(34)$ \\
Active malignancy & $15(7)$ \\
Reporting dyspnoea & $99(46)$ \\
Smoking & \\
Current & $38(18)$ \\
Former & $52(24)$ \\
Ongoing AC treatment & $81(38)$ \\
6MWT, mean (SD) & \\
Total & $449(135)$ \\
Men & $488(124)$ \\
Women & $402(134)$ \\
Laboratory tests at study visit & 203 \\
BNP, mean (SD) & $48.6(72.4)$ \\
F-score & 192 \\
Median (range) & $3(1-4)$ \\
\hline $6 M W T, 6$ min walking test measured in metres; & BMI, body mass \\
index (kg/m²), obesity=BMl>30 kg/m²; BNP, brain natriuretic \\
peptide (mg/l); F-score, Fredrikstad radiological score (higher \\
scores associated with a more proximal location of the thrombus); \\
Ongoing AC treatment, ongoing anticoagulant treatment at \\
inclusion; PE, pulmonary embolism; PE+DVT, concomitant deep \\
vein thrombosis reported in hospital records at PE diagnosis; \\
Unemployed, unemployed or unemployment because of long-term \\
illness or disability retirement; Working, working or studying. \\
\end{tabular}

The differences in mean EQ-5D index values were 0.11 and 0.06 between patients and buddy controls and between patients and the general population, respectively.

\section{Predictors of $\mathrm{HRQOL}$}

Table 4 summarises the results of the univariate analysis. The 6 min walking test was significantly correlated with all the EQ-5D dimensions as well as EQ VAS $(p<0.005)$, indicating that patients with lower scores on EQ VAS or reporting problems in the EQ-5D tended to walk shorter distances. Similar associations were found concerning dyspnoea, as those reporting dyspnoea reported problems in four out of five EQ-5D dimensions $(p<0.05$; table 4). Patients reporting dyspnoea also tended to cover shorter distances on the 6 min walking test (481 vs $413 \mathrm{~m}, \mathrm{p}<0.005)$. In the multiple linear regression model, the following variables were shown to be independently predictive of the dependent variable $\mathrm{EQ}$ VAS: performance on 6 min walking test $(\beta=0.09$, $\mathrm{p}<0.005)$, symptoms of dyspnoea $(\beta=-11.27, \mathrm{p}<0.005)$ and unemployment $(\beta=-8.98, \mathrm{p}<0.005$; table 5$)$. In addition to the EQ VAS, performance on the 6 min walking test consistently proved to be an independent determinant of every EQ-5D dimension, except for the dimension anxiety and depression. Dyspnoea was a significant predictor of the dimension usual activities and pain and discomfort. However, regarding the latter, the goodness of fit of the model showed a value beneath the significance level of 0.05 (Hosmer and Lemeshow=0.02), indicating the poor fit of the model. None of the other evaluated variables were significant determinants of HRQoL. The results from both the multiple linear and binary logistic regression analyses are displayed in table 5 .

\section{DISCUSSION}

In this population-based cross-sectional study, we found that the long-term HRQoL assessed by EQ-5D-3L was significantly impaired among patients with PE compared with buddy controls and population norms. Moreover, we found that poorer performance on the $6 \mathrm{~min}$ walking test, persistent patient-reported dyspnoea and unemployment were independent predictors of reduced HRQoL. To the best of our knowledge, this is the second largest study to compare long-term HRQoL after $\mathrm{PE}$ to a control group and the first one to incorporate a validated functional capacity test to a more comprehensive evaluation of HRQoL in patients with PE. Despite using a different instrument (EQ-5D-3L vs Short-Form 36 (SF-36)), our results of impaired HRQoL after PE confirm previously published studies. ${ }^{6}{ }^{7}$

The challenge of QoL studies is to judge whether identified differences are clinically relevant or not. Across various HRQoL research papers using the EQ-5D instrument, authors have suggested threshold values for minimal (clinical) important difference (MID/MCID), that is, the least amount of difference suggesting clinical relevance or mandating a change in clinical practice, ${ }^{22}$ ranging from 0.04 to $0.08 .^{23-25}$ In our study, the $\delta$ EQ-5D index value between the study population and buddy controls was 0.11 and between the study population and the general population was 0.06 . This indicates that we have identified the clinically relevant difference in HRQoL between the patients and both control cohorts. Of note, the cut-off value for MID/MCID is various and probably depends on the disease and valuation sets used. Moreover, since we did not include a longitudinal within-person measurement of QoL, the differences must be interpreted with caution. ${ }^{26}$

Compared with those without dyspnoea, $46.5 \%$ of our study patients who reported persistent dyspnoea performed worse in the 6 min walking test, objectively verifying this symptom. As for the whole study cohort, both male and female patients walked significantly shorter distances than their gender predicted value. In the 
Table 2 Frequency of patients $(n=213)$ reporting problems in the $E Q-5 D$ dimensions, means and medians for EQ VAS and $E Q-5 D$ index values. All displayed by age groups

\begin{tabular}{|c|c|c|c|c|c|c|c|c|}
\hline & \multicolumn{8}{|l|}{ Age groups } \\
\hline & 18-29 & 30-39 & $40-49$ & 50-59 & $60-69$ & $70-79$ & $80+$ & Total \\
\hline \multicolumn{9}{|l|}{ EQ-5D } \\
\hline Mobility, N (\%) & $2(1)$ & $4(2)$ & $6(3)$ & $8(4)$ & $12(6)$ & $13(6)$ & $10(5)$ & $55(26)$ \\
\hline Self-care, N (\%) & $0(0)$ & $0(0)$ & $2(1)$ & $3(1)$ & $3(1)$ & $5(2)$ & $1(0.5)$ & $14(7)$ \\
\hline $\begin{array}{l}\text { Usual activities, } \\
\mathrm{N}(\%)\end{array}$ & $2(1)$ & $4(2)$ & $9(4)$ & $10(5)$ & $20(9)$ & $11(5)$ & $12(6)$ & $68(32)$ \\
\hline $\begin{array}{l}\text { Pain and } \\
\text { discomfort, N (\%) }\end{array}$ & $3(1)$ & $8(4)$ & $15(7)$ & $21(10)$ & $28(13)$ & $24(11)$ & $17(8)$ & $116(54)$ \\
\hline $\begin{array}{l}\text { Anxiety and } \\
\text { depression, } \mathrm{N}(\%)\end{array}$ & $3(1)$ & $4(2)$ & $9(4)$ & $8(4)$ & $23(11)$ & $13(6)$ & $10(5)$ & $70(33)$ \\
\hline \multicolumn{9}{|l|}{ EQ VAS } \\
\hline Mean (SD) & $61(22)$ & $65(23)$ & $67(23)$ & $70(22)$ & $70(21)$ & $70(18)$ & $57(21)$ & $67(21)$ \\
\hline Median & 60 & 73 & 70 & 75 & 70 & 70 & 51 & 70 \\
\hline 25th & 45 & 49 & 50 & 50 & 53 & 51 & 40 & 50 \\
\hline 75th & 80 & 84 & 85 & 87 & 90 & 82 & 75 & 83 \\
\hline \multicolumn{9}{|l|}{ EQ-5D index values } \\
\hline Mean (SD) & $0.67(0.40)$ & $0.81(0.26)$ & $0.81(0.23)$ & $0.84(0.21)$ & $0.81(0.16)$ & $0.80(0.25)$ & $0.74(0.18)$ & $0.80(0.22)$ \\
\hline Median & 0.27 & 0.73 & 0.76 & 0.77 & 0.71 & 0.73 & 0.71 & 0.72 \\
\hline 25th & 0.82 & 0.82 & 0.82 & 0.82 & 0.79 & 0.82 & 0.77 & 0.82 \\
\hline 75th & 1.00 & 1.00 & 1.00 & 1.00 & 1.00 & 1.00 & 0.82 & 1.00 \\
\hline
\end{tabular}

Figure 2 Proportion of patients, Danish population and buddy controls reporting problems stratified by EQ-5D dimensions. EQ-5D, EuroQol five-dimension.

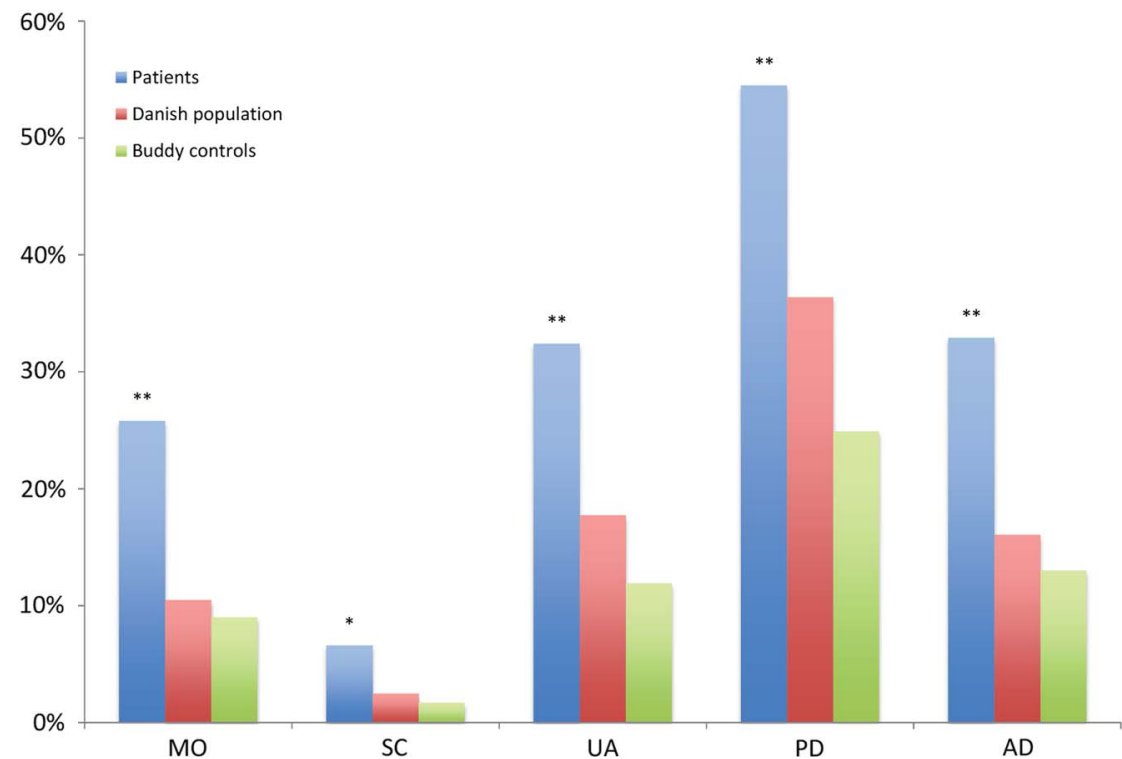

MO: Mobility, SC: Self-care, UA: Usual Activities, PD: Pain and Discomfort, AD: Anxiety and Depression ${ }^{*} \mathrm{p}<0.05 *{ }^{*} \mathrm{p}<0.005$, Chi-square, two-sided, patients vs. control groups. multivariable analyses, performance on the $6 \mathrm{~min}$ walking test and persistent dyspnoea appeared to be independent predictors of worse HRQoL. This may indicate that patients with PE suffer from a reduced functional capacity that persists for many years after the event and that the declining functional capacity is one of the main determinants of impaired HRQoL in patients with a history of PE. The finding that patients on average underperformed in the 6 min walking test may thus be an important explanation for their overall reduced HRQoL. This finding could be further supported by a qualitative study in patients with PE revealing that modification of physical activity and exertion (avoidance or reduction) was the most common behaviour change reported by the interviewed patients. Again, however, cut-off values for the clinically relevant abnormal 6 min walking test performance regarding PE are lacking, which makes it difficult to put the observed results in further perspective. Furthermore, we cannot exclude that this correlation also could be reversed, 
Table 3 Comparisons of mean EQ-5D index values and EQ VAS between patients versus Danish population and patients versus buddy controls

\begin{tabular}{|c|c|c|c|c|c|c|c|}
\hline & \multicolumn{3}{|l|}{ Patients } & \multicolumn{3}{|c|}{ Danish population* } & \multirow{2}{*}{$\begin{array}{l}\text { Buddy controls } \\
\text { Total }\end{array}$} \\
\hline & Male & Female & Total & Male & Female & Total & \\
\hline \multicolumn{8}{|l|}{ EQ-5D index } \\
\hline $\begin{array}{l}\text { Mean (SD) } \\
\text { p Value }\end{array}$ & $0.85(0.21)$ & $0.75(0.23)$ & $0.80(0.22)$ & $\begin{array}{l}0.87 \\
0.13 \dagger\end{array}$ & $\begin{array}{l}0.84 \\
<0.005 \dagger\end{array}$ & $\begin{array}{l}0.86 \\
<0.005 \dagger\end{array}$ & $\begin{array}{l}0.91(0.16) \\
<0.005 \ddagger\end{array}$ \\
\hline EQ VAS & & & & & & & \\
\hline $\begin{array}{l}\text { Mean (SD) } \\
\text { p Value }\end{array}$ & $71(20)$ & $62(22)$ & $67(21)$ & $\begin{array}{l}81 \\
<0.005 \dagger\end{array}$ & $\begin{array}{l}80 \\
<0.005 \dagger\end{array}$ & $\begin{array}{l}81 \\
<0.005 \dagger\end{array}$ & $\begin{array}{l}80(19) \\
<0.005 \ddagger\end{array}$ \\
\hline
\end{tabular}

${ }^{*}$ Age-adjusted and sex-adjusted values.

†One sample t-test with the age-adjusted and sex-adjusted value as test value (two-sided).

¥Mann-Whitney U test (two-sided).

EQ-5D, EuroQol five-dimension; EQ VAS, EuroQol visual analogue scale.

meaning that the reduced HRQoL is due to the low physical performance.

Numerous studies have shown the beneficial effects of pulmonary rehabilitation in other cardiovascular diseases, resulting in improved functional capacity as well as HRQoL. ${ }^{27-30}$ In this context, our findings support the hypothesis that patients with PE with persistent dyspnoea and poor functional status may benefit from cardiopulmonary rehabilitation programmes. ${ }^{31}$

Although the majority of studies focusing on the longterm effects of PE have not excluded patients with established CTEPH diagnosis, there is consistent reporting that approximately half of the patients assessed more than 6 months after experiencing an episode of acute $\mathrm{PE}$ report dyspnoea, which is also correlated to a decline in physical performance measured by the 6 min walking test. ${ }^{4} 1032 \quad 33$ Our results confirm these findings and support the concept of 'post-PE syndrome', which has recently been presented as an analogy to PTS, referring to the persistent dyspnoea and reduced functional capacity after PE. ${ }^{11}$ The authors discuss whether PE could in some cases, CTEPH excluded, be classified as a chronic illness and postulate the 'post-PE syndrome' being a state just prior to development of CTEPH. This reasoning is further strengthened by a Danish study reporting the overuse of antidepressants in adolescents long after they experienced their first episode of PE. ${ }^{12}$ However, whether persistent dyspnoea after PE should be the subject of further standardised workup including HRQoL questionnaires and the 6 min walking test is still debatable since some studies attribute the high prevalence of dyspnoea to pre-existing comorbidities. ${ }^{34}$ The final independent predictor of worse HRQoL in our study was unemployment. Several sociodemographic variables have previously been shown to affect HRQoL, regardless of the underlying disease or condition. ${ }^{35}$ The fact that $24 \%$ of the study population were unemployed could possibly have contributed to the overall lower HRQoL scores in our patient cohort. Furthermore, we hypothesise that the association between impaired HRQoL and unemployment may be subject to reverse correlation, that is, impaired HRQoL leading to unemployment. However, owing to missing data on other social factors, we could not investigate this further.

Of the predefined determinants being evaluated, we found only a selected proportion predictive of worse HRQoL and, to our surprise, malignancy appeared not to be a significant determinant of HRQoL. Previous studies have found cardiopulmonary disease, active malignancy as well as obesity being independent predictors of HRQoL. ${ }^{6}{ }^{36}$ However, the proportions of these subgroups reported in the aforementioned studies are higher than in ours and the presented multivariate regression analysis yielded rather low $r^{2}$ percentages indicating the models not being precise. ${ }^{6}$ This may indicate, as van Es $e t a l^{7}$ postulate, that in this study the patients are somewhat healthier and subsequently, perhaps emphasising the findings regarding the reported differences in HRQoL between study participants and the general population. Nevertheless, in our view, these contradictions exemplify the heterogeneous effects of $\mathrm{PE}$ as a disease on HRQoL and physical capacity and consequently rendering cumbersome the evaluation of determinants of HRQoL in patients with PE.

\section{Limitations}

Our study has some limitations. The low response rate may hamper the external validity of our results. Moreover, since the final study cohort comprised onequarter of the patients being assessed for eligibility, a possible bias towards recruitment of patients with more persistent symptoms cannot be ruled out. However, the 6 min walking test results and proportion reporting dyspnoea in our sample are similar to those of prior PE follow-up studies, ${ }^{10} 33{ }^{37}$ highlighting that our cohort is a representative PE population. Also, the buddy control group could not be assessed for potential confounders because we did not assess their characteristics. Therefore, we cannot rule out a bias towards 'extremely' healthy buddies or poor matching. Furthermore, owing to the study's retrospective design, which carried missing data concerning the index event of the PE, we could not classify the PE episode as being of low or intermediate risk. 
Table 4 Univariate analysis displaying correlations of the predefined determinants to EQ-5D dimensions and EQ VAS

\begin{tabular}{|c|c|c|c|c|c|c|c|c|c|c|c|c|c|}
\hline & \multirow[b]{3}{*}{$\mathbf{N}$} & \multicolumn{10}{|l|}{ EQ-5D } & \multicolumn{2}{|l|}{ EQ VAS } \\
\hline & & \multicolumn{2}{|l|}{ MO } & \multicolumn{2}{|l|}{ SC } & \multicolumn{2}{|l|}{ UA } & \multicolumn{2}{|l|}{ PD } & \multicolumn{2}{|l|}{ AD } & \multirow[b]{2}{*}{ Corr.coef } & \multirow[b]{2}{*}{ p Value } \\
\hline & & Corr.coef & p Value & Corr.coef & p Value & Corr.coef & p Value & Corr.coef & p Value & Corr.coef & p Value & & \\
\hline Age & 213 & 0.09 & 0.19 & 0.05 & 0.49 & 0.07 & 0.32 & 0.06 & 0.39 & 0.06 & 0.38 & -0.06 & 0.31 \\
\hline Sex & 213 & $-0.20^{\star}$ & $<0.05$ & -0.03 & 0.70 & $-0.23^{*}$ & $<0.05$ & $-0.13^{\star}$ & 0.06 & $-0.27^{\star}$ & $<0.05$ & $0.23^{\star}$ & $<0.05$ \\
\hline 6MWT & 213 & $-0.43^{*}$ & $<0.05$ & $-0.28^{\star}$ & $<0.05$ & $-0.42^{\star}$ & $<0.05$ & $-0.30^{\star}$ & $<0.05$ & $-0.25^{\star}$ & $<0.05$ & $0.51^{*}$ & $<0.05$ \\
\hline BMI & 213 & $0.13^{\star}$ & 0.06 & $0.14^{\star}$ & 0.04 & 0.10 & 0.16 & 0.03 & 0.71 & 0.05 & 0.50 & $0.22^{*}$ & $<0.05$ \\
\hline BNP & 203 & $0.19^{*}$ & 0.01 & 0.01 & 0.89 & $0.15^{\star}$ & 0.03 & $0.13^{*}$ & 0.07 & $0.19^{*}$ & 0.01 & -0.10 & 0.14 \\
\hline Ongoing AC & 213 & 0.09 & 0.19 & -0.05 & 0.45 & 0.02 & 0.73 & 0.02 & 0.80 & -0.01 & 0.85 & -0.06 & 0.38 \\
\hline Cardiopulmonary comorbidity & 213 & $0.15^{\star}$ & 0.03 & 0.05 & 0.47 & $0.17^{*}$ & 0.01 & 0.09 & 0.20 & 0.06 & 0.20 & $-0.19^{\star}$ & 0.01 \\
\hline Active cancer & 213 & 0.09 & 0.20 & 0.01 & 0.99 & 0.01 & 0.90 & -0.01 & 0.93 & 0.01 & 0.97 & -0.02 & 0.77 \\
\hline Reporting dyspnoea & 213 & $0.16^{*}$ & 0.02 & $0.13^{*}$ & 0.05 & $0.29^{*}$ & $<0.05$ & $0.40^{*}$ & $<0.05$ & 0.11 & 0.11 & $-0.37^{*}$ & $<0.05$ \\
\hline F-score & 192 & 0.06 & 0.42 & $0.15^{\star}$ & 0.04 & 0.11 & 0.13 & 0.07 & 0.36 & -0.01 & 0.94 & $-0.12^{*}$ & 0.09 \\
\hline Recurrent VTE & 213 & 0.01 & 0.93 & -0.06 & 0.35 & -0.02 & 0.73 & 0.06 & 0.35 & -0.01 & 0.95 & -0.02 & 0.77 \\
\hline Unemployed & 213 & 0.10 & 0.13 & 0.08 & 0.27 & $0.22^{*}$ & $<0.05$ & $0.24^{*}$ & $<0.05$ & $0.27^{*}$ & $<0.05$ & $-0.24^{*}$ & 0.01 \\
\hline Disease duration & 213 & -0.02 & 0.77 & -0.02 & 0.79 & -0.03 & 0.64 & -0.04 & 0.58 & -0.07 & 0.32 & $0.13^{*}$ & 0.05 \\
\hline
\end{tabular}

${ }^{*}$ All values with $\alpha<0.10$ retained for multiple regression analysis, explanatory variables recoded to $0=$ not having the condition and $1=$ having the condition, dimensions dichotomised in reporting problems $=1$ and not reporting problems $=0$.

6MWT, 6 min walking test; AD, anxiety and depression; Age, age at inclusion, male sex=1; BMI, body mass index $\left(\mathrm{kg} / \mathrm{m}^{2}\right)$; BNP, brain natriuretic peptide; Corr. coef, Spearman's $\mathrm{r}$ correlation coefficient; EQ-5D, EuroQol five-dimension; EQ VAS, EuroQol visual analogue scale; F-score, Fredrikstad radiological score (higher scores associated with a more proximal location of the

thrombus); MO, mobility; Ongoing AC, ongoing anticoagulant treatment; PD, pain and discomfort; SC, self-care; UA, usual activities; Unemployed, unemployed or unemployment because of long-term illness or disability retirement and disease duration=time in years from PE diagnosis to study inclusion. 
Table 5 Multiple binary logistic and standard linear regression models with retained determinants from the univariate analysis for possible independency tested against EQ-5D dimensions and EQ VAS

\begin{tabular}{|c|c|c|c|c|c|c|c|c|c|c|c|c|}
\hline & \multicolumn{10}{|c|}{ EQ-5D } & \multicolumn{2}{|l|}{ EQ VAS } \\
\hline & \multicolumn{2}{|l|}{ MO } & \multicolumn{2}{|l|}{ SC } & \multicolumn{2}{|l|}{ UA } & \multicolumn{2}{|l|}{ PD } & \multicolumn{2}{|l|}{ AD } & \multirow[b]{2}{*}{$\beta \dagger$} & \multirow[b]{2}{*}{ SE‡ } \\
\hline & $\overline{\text { OR }}$ & $95 \% \mathrm{Cl}$ & $\overline{\text { OR }}$ & $95 \% \mathrm{Cl}$ & $\overline{\text { OR }}$ & $95 \% \mathrm{Cl}$ & $\overline{\text { OR }}$ & $95 \% \mathrm{Cl}$ & $\overline{\text { OR }}$ & $95 \% \mathrm{Cl}$ & & \\
\hline 6MWT & 0.991 & 0.987 to $0.995^{\star \star}$ & 0.990 & 0.984 to $0.997^{* \star}$ & 0.991 & 0.988 to $0.995^{\star \star}$ & 0.996 & 0.992 to $0.999^{*}$ & 0.997 & 0.994 to 1.000 & 0.09 & $0.01^{* \star}$ \\
\hline $\mathrm{BMI}$ & 1.04 & 0.97 to 1.12 & 1.06 & 0.94 to 1.19 & - & - & - & - & - & - & -0.07 & 0.26 \\
\hline BNP & 1.00 & 1.00 to 1.01 & - & - & 1.00 & 1.00 to 1.01 & 1.00 & 0.99 to 1.00 & 1.00 & 1.00 to 1.01 & - & - \\
\hline Cardiopulmonary comorbidity & 0.57 & 0.14 to 2.30 & - & - & 0.78 & 0.20 to 3.07 & - & - & - & - & 1.63 & 4.69 \\
\hline Reporting dyspnoea & 1.33 & 0.63 to 2.81 & 1.74 & 0.38 to 8.07 & 2.33 & 1.14 to $4.78^{*}$ & 3.74 & 1.97 to $7.08^{\star *}$ & - & - & -11.27 & $2.56^{\star *}$ \\
\hline F-score & - & - & 3.12 & 0.79 to 5.69 & - & - & - & - & - & - & 1.71 & 1.13 \\
\hline Unemployed & - & - & - & - & 2.55 & 1.15 to $5.67^{*}$ & 2.76 & 1.24 to $6.15^{*}$ & 3.94 & 1.88 to $8.26^{\star *}$ & -8.98 & $2.97^{* *}$ \\
\hline Disease duration & - & - & - & - & - & - & - & - & - & - & -0.19 & 0.47 \\
\hline $\begin{array}{l}\text { Hosmer and Lemeshow } \\
r^{2}\end{array}$ & 0.08 & & 0.87 & & 0.35 & & 0.02 & & 0.62 & & 0.46 & \\
\hline
\end{tabular}

${ }^{*} p$ Value $<0.05$ all regression models adjusted for age and sex, explanatory variables recoded to $0=$ not having the condition and $1=$ having the condition, dimensions dichotomised in reporting problems $=1$ and not reporting problems $=0$.

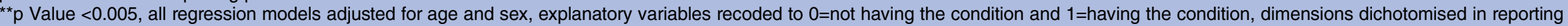
problems $=1$ and not reporting problems $=0$.

Unstandardised $\beta$ coefficient.

¥SE of $\beta$.

6MWT, 6 min walking test; $A D$, anxiety and depression, higher scores in EQ VAS associated with better health related quality of life; BMI, body mass index ( $\mathrm{kg} / \mathrm{m}^{2}$ ); $\mathrm{BNP}$, brain natriuretic peptide; EQ-5D, EuroQol five-dimension; EQ VAS, EuroQol visual analogue scale; F-score, Fredrikstad radiological score (higher scores associated with a more proximal location of the thrombus); MO, mobility; PD, pain and discomfort; SC, self-care; UA, usual activities; Unemployed, unemployed or unemployment because of long-term illness or disability retirement, disease duration=time in years from diagnosis to study inclusion. 
The EQ-5D was used based on its simplicity and potential positive influence on patients' completeness of scores. It could be argued, however, that the SF-36 might have been a good choice as well, due to its comprehensiveness and in order to compare our results with previous studies. Finally, we did not apply a disease-specific questionnaire of QoL.

The strong points of this study are the sample size and the long-term follow-up period with $89 \%$ of the patients being diagnosed with $\mathrm{PE}$ more than 1 year prior to inclusion. Furthermore, this study is one of the largest studies to present a more comprehensive evaluation of HRQoL by reporting all aspects of a generic QoL questionnaire as well as incorporating a functional capacity test (6 min walking test) in order to objectify the findings.

\section{CONCLUSIONS}

Patients with a history of acute PE were found to have a worse HRQoL compared with age-matched and sexmatched venous thromboembolism-free buddy controls and population controls. Underperformance and patient-reported dyspnoea were independent predictors of decreased HRQoL. Further studies are necessary to further evaluate the course and determinants of HRQoL after acute PE, as well as interventions aimed at improving HRQoL in these patients.

Contributors MT and WG were responsible for study concept, design and data acquisition. MT, HSW and WG performed the statistical analyses. MT wrote the first draft of the manuscript. All authors were responsible for critical revision of the manuscript, interpretation of the results, had full access to all the data in the study, and take responsibility of the integrity of the data and the accuracy of data analysis.

Funding This research received no specific grant from any funding agency in the public, commercial or not-for-profit sectors.

Competing interests WG reports grants and lecture honoraria from Bayer, Novartis and Roche and lecture and advisory board honoraria from Pfizer, Bayer and from Boehringer Ingelheim. LPJ-J reports unrestricted grants from Ferring pharmaceuticals and Tillots pharma, and personal fees from Abbvie.

Ethics approval The study was approved by the Regional Committee for Medical and Health Research Ethics, Norway (Approval no 2011/2557b).

Provenance and peer review Not commissioned; externally peer reviewed.

Data sharing statement No additional data are available.

Open Access This is an Open Access article distributed in accordance with the Creative Commons Attribution Non Commercial (CC BY-NC 4.0) license, which permits others to distribute, remix, adapt, build upon this work noncommercially, and license their derivative works on different terms, provided the original work is properly cited and the use is non-commercial. See: http:// creativecommons.org/licenses/by-nc/4.0/

\section{REFERENCES}

1. Kahn SR. The post-thrombotic syndrome: progress and pitfalls. $\mathrm{Br} J$ Haematol 2006;134:357-65.

2. Fedullo PF, Auger WR, Kerr KM, et al. Chronic thromboembolic pulmonary hypertension. N Engl J Med 2001;345:1465-72.

3. Pengo V, Lensing AWA, Prins $\mathrm{MH}$, et al. Incidence of chronic thromboembolic pulmonary hypertension after pulmonary embolism. N Engl J Med 2004;350:2257-64.
4. Klok FA, Tijmensen JE, Haeck MLA, et al. Persistent dyspnea complaints at long-term follow-up after an episode of acute pulmonary embolism: results of a questionnaire. Eur $\mathrm{J}$ Intern Med 2008;19:625-9.

5. Klok FA, Cohn DM, Middeldorp S, et al. Quality of life after pulmonary embolism: validation of the PEmb-QoL Questionnaire. $J$ Thromb Haemost 2010;8:523-32.

6. Klok FA, van Kralingen KW, van Dijk APJ, et al. Quality of life in long-term survivors of acute pulmonary embolism. Chest 2010;138:1432-40.

7. van Es J, den Exter PL, et al Quality of life after pulmonary embolism as assessed with SF-36 and PEmb-QoL. Thromb Res 2013;132:500-5.

8. Noble S, Lewis R, Whithers $\mathrm{J}$, et al. Long-term psychological consequences of symptomatic pulmonary embolism: a qualitative study. BMJ Open 2014;4:e004561.

9. Bennett P, Patterson K, Noble S. Predicting post-traumatic stress and health anxiety following a venous thrombotic embolism. J Health Psychol 2016;21:863-71.

10. Kline JA, Steuerwald MT, Marchick MR, et al. Prospective evaluation of right ventricular function and functional status 6 months after acute submassive pulmonary embolism. Chest 2009:136:1202-10.

11. Klok FA, van der Hulle T, Exter den PL, et al. The post-PE syndrome: a new concept for chronic complications of pulmonary embolism. Blood Rev 2014;28:221-6.

12. Højen AA, Gorst-Rasmussen A, Lip GYH, et al. Use of psychotropic drugs following venous thromboembolism in youth. A nationwide cohort study. Thromb Res 2015;135:643-7.

13. Rasekaba T, Lee AL, Naughton MT, et al. The six-minute walk test: a useful metric for the cardiopulmonary patient. Intern Med $J$ 2009;39:495-501.

14. ATS Committee on Proficiency Standards for Clinical Pulmonary Function Laboratories. ATS statement: guidelines for the six-minute walk test. Am J Respir Crit Care Med 2002;166: 111-17.

15. Enright PL, Sherrill DL. Reference equations for the six-minute walk in healthy adults. Am J Respir Crit Care Med 1998;158: 1384-7.

16. EuroQol Group. EuroQol-a new facility for the measurement of health-related quality of life. Health Policy 1990;16:199-208.

17. Brooks R. EuroQol: the current state of play. Health Policy 1996;37:53-72.

18. Wittrup-Jensen KU, Lauridsen J, Gudex C, et al. Generation of a Danish TTO value set for EQ-5D health states. Scand J Public Health 2009;37:459-66.

19. Ghanima W, Abdelnoor M, Holmen LO, et al. The association between the proximal extension of the clot and the severity of pulmonary embolism (PE): a proposal for a new radiological score for PE. J Intern Med 2007;261:74-81.

20. Hjermstad MJ, Fayers PM, Bjordal K, et al. Using reference data on quality of life-the importance of adjusting for age and gender, exemplified by the EORTC QLQ-C30 (+ 3). Eur J Cancer 1998;34:1381-9.

21. Simons CL, Rivero-Arias O, Yu LM, et al. Multiple imputation to deal with missing EQ-5D-3L data: Should we impute individual domains or the actual index? Qual Life Res 2015;24:805-15.

22. Jaeschke R, Singer J, Guyatt GH. Measurement of health status. Ascertaining the minimal clinically important difference. Control Clin Trials 1989;10:407-15.

23. Marra CA, Woolcott JC, Kopec JA, et al. A comparison of generic, indirect utility measures (the HUI2, HUI3, SF-6D, and the EQ-5D) and disease-specific instruments (the RAQoL and the HAQ) in rheumatoid arthritis. Soc Sci Med 2005;60:1571-82.

24. Walters SJ, Brazier JE. Comparison of the minimally important difference for two health state utility measures: EQ-5D and SF-6D. Qual Life Res 2005;14:1523-32.

25. Luo N, Johnson J, Coons SJ. Using instrument-defined health state transitions to estimate minimally important differences for four preference-based health-related quality of life instruments. Med Care 2010;48:365-71.

26. de Vet HCW, Ostelo RWJG, Terwee CB, et al. Minimally important change determined by a visual method integrating an anchorbased and a distribution-based approach. Qual Life Res 2007;16: 131-42.

27. Ehlken $\mathrm{N}$, Lichtblau $\mathrm{M}$, Klose $\mathrm{H}$, et al. Exercise training improves peak oxygen consumption and haemodynamics in patients with severe pulmonary arterial hypertension and inoperable chronic thrombo-embolic pulmonary hypertension: a prospective, randomized, controlled trial. Eur Heart $J$ 2016;37:35-44. 
28. Nagel C, Prange F, Guth S, et al. Exercise training improves exercise capacity and quality of life in patients with inoperable or residual chronic thromboembolic pulmonary hypertension. PLoS ONE 2012;7:e41603-9.

29. Rochester CL, Fairburn C, Crouch $\mathrm{RH}$. Pulmonary rehabilitation for respiratory disorders other than chronic obstructive pulmonary disease. Clin Chest Med 2014;35:369-89.

30. Yuan $\mathrm{P}$, Yuan $\mathrm{XT}$, Sun $\mathrm{XY}$, et al. Exercise training for pulmonary hypertension: a systematic review and meta-analysis. Int J Cardiol 2015;178:142-6.

31. Lakoski SG, Savage PD, Berkman AM, et al. The safety and efficacy of early-initiation exercise training after acute venous thromboembolism: a randomized clinical trial. J Thromb Haemost 2015;13:1238-44.

32. Stevinson BG, Hernandez-Nino J, Rose G, et al. Echocardiographic and functional cardiopulmonary problems 6 months after first-time pulmonary embolism in previously healthy patients. Eur Heart $J$ 2007;28:2517-24.
33. Chow V, Ng ACC, Seccombe L, et al. Impaired 6-min walk test, heart rate recovery and cardiac function post pulmonary embolism in long-term survivors. Respir Med 2014;108:1556-65.

34. Klok FA, van Kralingen KW, van Dijk APJ, et al. Prevalence and potential determinants of exertional dyspnea after acute pulmonary embolism. Respir Med 2010;104:1744-9.

35. Grandy S, Fox KM. EQ-5D visual analog scale and utility index values in individuals with diabetes and at risk for diabetes: findings from the Study to Help Improve Early evaluation and management of risk factors Leading to Diabetes (SHIELD). Health Qual Life Outcomes 2008;6:18.

36. Stewart LK, Peitz GW, Nordenholz KE, et al. Contribution of fibrinolysis to the physical component summary of the SF-36 after acute submassive pulmonary embolism. J Thromb Thrombolysis 2015;40:161-6.

37. Sanchez O, Helley D, Couchon S, et al. Perfusion defects after pulmonary embolism: risk factors and clinical significance. $J$ Thromb Haemost 2010;8:1248-55. 